\title{
Computational Results of Duadic Double Circulant Codes
}

\author{
Sunghyu Han \\ School of Liberal Arts \\ Korea University of Technology and Education \\ Cheonan 330-708, S. Korea \\ Email: sunghyu@kut.ac.kr \\ and \\ Jon-Lark Kim * \\ Department of Mathematics \\ University of Louisville \\ Louisville, KY 40292, USA \\ Email: jl.kim@louisville.edu
}

\begin{abstract}
Quadratic residue codes have been one of the most important classes of algebraic codes. They have been generalized into duadic codes and quadratic double circulant codes. In this paper we introduce a new subclass of double circulant codes, called duadic double circulant codes, which is a generalization of quadratic double circulant codes for prime lengths. This class generates optimal self-dual codes, optimal linear codes, and linear codes with the best known parameters in a systematic way. We describe a method to construct duadic double circulant codes using 4-cyclotomic cosets and give certain duadic double circulant codes over $\mathbb{F}_{2}, \mathbb{F}_{3}, \mathbb{F}_{4}, \mathbb{F}_{5}$, and $\mathbb{F}_{7}$. In particular, we find a new ternary self-dual $[76,38,18]$ code and easily rediscover optimal binary self-dual codes with parameters $[66,33,12],[68,34,12],[86,43,16]$, and $[88,44,16]$ as well as a formally self-dual binary $[82,41,14]$ code.
\end{abstract}

Key Words: Double circulant codes, duadic codes, duadic double circulant codes, quadratic reside codes.

AMS subject classification: 94B05, 11T71, 05E99

*corresponding author 


\section{Introduction}

Quadratic Residue (QR) codes have been one of the most interesting classes of linear codes. They have a good decoding algorithm called permutation decoding, thanks to a large automorphism group. They also have high minimum distances and sometimes are self-dual.

On the other hand, M. Karlin [17] considered binary double circulant codes based on quadratic residues. Later, V. Pless [22] considered ternary double circulant codes based on quadratic residues, producing the famous Pless symmetry codes. She also introduced a class of duadic codes as a natural generalization of QR codes. Duadic codes were generalized into triadic codes [23], polyadic codes $([1],[20])$ and duadic codes [19] over $\mathbb{Z}_{4}$. P. Gaborit [5] introduced quadratic double circulant codes which included Karlin's above construction and the Pless symmetry codes. He constructed new infinite families of self-dual codes over $G F(4), G F(5), G F(7)$, and $G F(9)$ [5]. A uniform generalization of quadratic double circulant codes was given by S. Dougherty, et. al. 4] using two class association schemes.

In this paper, we introduce a new subclass of double circulant codes, called duadic double circulant codes. This is a generalization of quadratic double circulant codes for prime lengths and also can produce non self-dual codes. This class generates optimal selfdual codes, optimal linear codes, and linear codes with the best known parameters in a systematic way. For another motivation of duadic double circulant codes, we observe that there has been a classification of extremal double circulant self-dual codes of lengths up to 88 (see [10], [11]). These codes are classified by an exhaustive computer search. Hence it is natural to construct a subclass of double circulant codes algebraically which contains both self-dual and non self-dual codes.

We compute all duadic double circulant codes of lengths up to 82,82,62,58,38 over $\mathbb{F}_{2}, \mathbb{F}_{3}, \mathbb{F}_{4}, \mathbb{F}_{5}$, and $\mathbb{F}_{7}$, respectively, that are based on 4 -cyclotomic cosets. In particular, we rediscover optimal binary self-dual codes with parameters $[66,33,12],[68,34,12],[86,43,16]$, and $[88,44,16]$ as well as a formally self-dual binary $[82,41,14]$ code. Using 9-cyclotomic cosets, we find a new ternary self-dual $[76,38,18]$ code. We further construct optimal formally self-dual codes or codes with the best known parameters, which cannot be obtained from quadratic double circulant construction (see Example 4.1 (iv) and Example 4.2).

\section{Splitting}

We begin with some definitions. Let $n$ be a positive integer and $a(1 \leq a \leq n-1)$ be an integer such that $\operatorname{gcd}(a, n)=1$. Then the function $\mu_{a}$ defined on $\{0,1,2, \cdots, n-1\}$ by $i \mu_{a} \equiv i a(\bmod n)$ is called a multiplier.

Definition 2.1. Let $n$ be an odd positive integer with $n>1$. A pair $\left(S_{1}, S_{2}\right)$ of two sets $S_{1}$ and $S_{2}$ is called a (generalized duadic) splitting of $n$ if the following two conditions are satisfied:

(i) $S_{1}$ and $S_{2}$ satisfy

$$
S_{1} \cup S_{2}=\{1,2, \cdots, n-1\} \text { and } S_{1} \cap S_{2}=\emptyset,
$$


(ii) there is a multiplier $\mu_{a}$ such that

$$
S_{1} \mu_{a}=S_{2} \text { and } S_{2} \mu_{a}=S_{1}
$$

In fact, condition (ii) of Definition 2.1 can be weakened as follows.

Lemma 2.2. Let $A=\{1,2, \cdots, n-1\}$, with odd $n \geq 1, S_{1} \cup S_{2}=A$, and $S_{1} \cap S_{2}=\emptyset$. Let $\mu_{a}$ be a multiplier. If $S_{1} \mu_{a}=S_{2}$, then $S_{2} \mu_{a}=S_{1}$.

Proof. Since $\mu_{a}$ is bijective on $A$, we have $A=A \mu_{a}=S_{1} \mu_{a} \cup S_{2} \mu_{a}$ and $S_{1} \mu_{a} \cap S_{2} \mu_{a}=\emptyset$. Hence if $S_{1} \mu_{a}=S_{2}$, then $S_{2} \mu_{a}=S_{1}$.

It is natural to ask when there exists a splitting of a given odd integer $n$.

Proposition 2.3. Let $\operatorname{ord}_{n}(a)$ denote the multiplicative order of a modulo $n$. Then the following hold.

(i) There exists a splitting for any odd $n$ with $n>1$.

(ii) If $\operatorname{ord}_{n}(a)$ is odd, then there is no splitting of $n$ with $\mu_{a}$.

(iii) If $n$ is a prime and $\operatorname{ord}_{n}(a)$ is $n-1$, then the splitting $S_{1}$ and $S_{2}$ is the set of quadratic residues and the set of quadratic nonresidues of $n$.

Proof. For (ii), we can take $S_{1}=\left\{1,2, \cdots, \frac{n-1}{2}\right\}$ and $S_{2}=\left\{\frac{n+1}{2}, \frac{n+3}{2}, \cdots, n-1\right\}$ with $\mu_{-1}$. For (iil) and (iii), without loss of generality, we may assume that $S_{1}$ contains 1 . Then $S_{1}=$ $\left\{1, a^{2}, a^{4}, \cdots\right\}$ and $S_{2}=\left\{a, a^{3}, a^{5}, \cdots\right\}$ under $\mu_{a}$ by Lemma 2.2. Suppose $\operatorname{ord}_{n}(a)=2 k+1$ for some $k$. Then $a^{2 k} \in S_{1}$ and $a^{2 k} \mu_{a}=1 \in S_{1}$. This is a contradiction. This proves (ii). Next suppose that $n$ is a prime and that $\operatorname{ord}_{n}(a)=n-1$. Then $S_{1}$ is the set of quadratic residues and $S_{2}$ is the set of quadratic nonresidues of $n$, proving (iii).

\section{Duadic double circulant codes}

Now we are ready to define Duadic Double Circulant (DDC) codes. First, we choose a splitting $\left(S_{1}, S_{2}\right)$ for an odd positive integer $n$, where $S_{1}$ and $S_{2}$ do not need to be unions of nonzero $q$-cyclotomic cosets. With this splitting, we define two generating matrices below.

$$
\begin{aligned}
P_{n}\left(r, s, t, S_{1}, S_{2}\right) & =\left(I \mid D_{n}\left(r, s, t, S_{1}, S_{2}\right)\right) \\
B_{n}\left(\alpha, \beta, \gamma, r, s, t, S_{1}, S_{2}\right) & =\left(\begin{array}{c|ccc|c|cc}
1 & 0 & \cdots & 0 & \alpha & \beta & \cdots \\
\hline 0 & & & \gamma & \\
\vdots & & I & \vdots & D_{n}\left(r, s, t, S_{1}, S_{2}\right) \\
0 & & & \gamma &
\end{array}\right),
\end{aligned}
$$

where $r, s, t, \alpha, \beta, \gamma \in \mathbb{F}_{q}, I$ is an $n \times n$ identity matrix, and $D_{n}\left(r, s, t, S_{1}, S_{2}\right)$ is an $n \times n$ circulant matrix whose first row is defined as follows. Suppose that $\left(a_{0}, a_{1}, \cdots, a_{n-1}\right)$ is the 
first row of $D_{n}\left(r, s, t, S_{1}, S_{2}\right)$. Then define $a_{0}=r, a_{i}=s$ if $i \in S_{1}$, and $a_{i}=t$ if $i \in S_{2}$. Using these two generating matrices, we define $[2 n, n]$ and $[2 n+2, n+1]$ linear codes over $\mathbb{F}_{q}$ and we denote them by $\mathcal{P}_{n}\left(r, s, t, S_{1}, S_{2}\right)$ and $\mathcal{B}_{n}\left(\alpha, \beta, \gamma, r, s, t, S_{1}, S_{2}\right)$ respectively. We call $\mathcal{P}_{n}\left(r, s, t, S_{1}, S_{2}\right)$ a duadic pure double circulant code, $\mathcal{B}_{n}\left(\alpha, \beta, \gamma, r, s, t, S_{1}, S_{2}\right)$ duadic bordered double circulant code, and we call both duadic double circulant codes. Note that duadic double circulant codes contain Gaborit's quadratic double circulant codes [5] when $n$ is a prime by Proposition 2.3 (iii). We also remark that $\mathcal{P}_{n}\left(r, s, t, S_{1}, S_{2}\right)$ over $\mathbb{F}_{2}$ is always formally self-dual [16, Sec. 9.8], while $\mathcal{B}_{n}\left(\alpha, \beta, \gamma, r, s, t, S_{1}, S_{2}\right)$ is not necessarily. (See Example 4.1 in Section 4.)

Proposition 3.1. The codes $\mathcal{P}_{n}\left(r, s, t, S_{1}, S_{2}\right)$ and $\mathcal{P}_{n}\left(r, s, t, S_{2}, S_{1}\right)$ are equivalent. Similarly, $\mathcal{B}_{n}\left(\alpha, \beta, \gamma, r, s, t, S_{1}, S_{2}\right)$ and $\mathcal{B}_{n}\left(\alpha, \beta, \gamma, r, s, t, S_{2}, S_{1}\right)$ are equivalent.

The proof of this proposition follows from the below lemmas.

Lemma 3.2. Let $\mathbb{F}_{q}$ be an arbitrary finite field. Let $\mathcal{R}_{n}=\mathbb{F}_{q}[x] /\left(x^{n}-1\right)$, $\operatorname{gcd}(n, q)=1$ and $\mu_{a}$ be a multiplier with $\operatorname{gcd}(a, n)=1$. Consider the following two pure double circulant codes in $\mathcal{R}_{n} \oplus \mathcal{R}_{n}$.

$$
\begin{aligned}
& C_{1}=\left\{\left(f(x), e_{1}(x) f(x)\right) \mid f(x) \in \mathcal{R}_{n}\right\}, \\
& C_{2}=\left\{\left(f(x), e_{2}(x) f(x)\right) \mid f(x) \in \mathcal{R}_{n}\right\},
\end{aligned}
$$

where $e_{2}(x)=e_{1}(x) \mu_{a}$. Then $C_{1}$ and $C_{2}$ are permutation equivalent.

Proof. Let

$$
C_{1} \mu_{a}=\left\{\left(f(x) \mu_{a},\left(e_{1}(x) f(x)\right) \mu_{a}\right) \mid f(x) \in \mathcal{R}_{n}\right\} .
$$

In [16, Theorem 4.3.12], we know that $\mu_{a}$ is an automorphism of $\mathcal{R}_{n}$. Therefore,

$$
\begin{aligned}
C_{1} \mu_{a} & =\left\{\left(f(x) \mu_{a},\left(e_{1}(x) \mu_{a}\right)\left(f(x) \mu_{a}\right)\right) \mid f(x) \in \mathcal{R}_{n}\right\} \\
& =\left\{\left(h(x), e_{2}(x) h(x)\right) \mid h(x) \in \mathcal{R}_{n}\right\} \\
& =C_{2}
\end{aligned}
$$

Lemma 3.3. Let $\mathbb{F}_{q}$ be an arbitrary finite field. Let $\mathcal{R}_{n}=\mathbb{F}_{q}[x] /\left(x^{n}-1\right) \operatorname{gcd}(n, q)=1$ and $\mu_{a}$ be a multiplier with $\operatorname{gcd}(a, n)=1$. Consider the following two bordered double circulant codes in $\mathbb{F}_{q} \oplus \mathcal{R}_{n} \oplus \mathbb{F}_{q} \oplus \mathcal{R}_{n}$.

$$
\begin{aligned}
& C_{1}=\left\{\left(b, f(x), b \alpha+f(1) \gamma, b \beta j(x)+e_{1}(x) f(x)\right) \mid b \in \mathbb{F}_{q}, f(x) \in \mathcal{R}_{n}\right\}, \\
& C_{2}=\left\{\left(b, f(x), b \alpha+f(1) \gamma, b \beta j(x)+e_{2}(x) f(x)\right) \mid b \in \mathbb{F}_{q}, f(x) \in \mathcal{R}_{n}\right\},
\end{aligned}
$$

where $\alpha, \beta, \gamma \in \mathbb{F}_{q}, e_{2}(x)=e_{1}(x) \mu_{a}, j(x)=1+x+x^{2}+\cdots+x^{n-1}$. Then $C_{1}$ and $C_{2}$ are permutation equivalent. 
Proof. Let

$$
C_{1} \mu_{a}=\left\{\left(b, f(x) \mu_{a}, b \alpha+f(1) \gamma,\left(b \beta j(x)+e_{1}(x) f(x)\right) \mu_{a} \mid b \in \mathbb{F}_{q}, f(x) \in \mathcal{R}_{n}\right\}\right.
$$

Then,

$$
\begin{aligned}
C_{1} \mu_{a} & =\left\{\left(b, f(x) \mu_{a}, b \alpha+f(1) \gamma, b \beta j(x)+\left(e_{1}(x) \mu_{a}\right)\left(f(x) \mu_{a}\right) \mid b \in \mathbb{F}_{q}, f(x) \in \mathcal{R}_{n}\right\}\right. \\
& =\left\{\left(b, h(x), b \alpha+h(1) \gamma, b \beta j(x)+e_{2}(x) h(x) \mid b \in \mathbb{F}_{q}, h(x) \in \mathcal{R}_{n}\right\} .\right. \\
& =C_{2} .
\end{aligned}
$$

Definition 3.4. Suppose that there is a splitting $\left(S_{1}, S_{2}\right)$ of $n$ and let $q$ be a power of a prime with $\operatorname{gcd}(q, n)=1$. Furthermore, if $S_{1}$ and $S_{2}$ are unions of nonzero $q$-cyclotomic cosets, then the cyclic codes with defining sets $S_{1}$ and $S_{2}$ are called the odd-like duadic codes of length $n$ over $\mathbb{F}_{q}$. On the other hand, the cyclic codes with defining sets $S_{1} \cup\{0\}$ and $S_{2} \cup\{0\}$ are called the even-like duadic codes of length $n$ over $\mathbb{F}_{q}$.

Theorem 3.5. [16, Chapter 6] Duadic codes of odd length $n$ over $\mathbb{F}_{q}$ exist if and only if $q$ is a square modulo $n$.

Thus we have a systematic way to construct splittings of $n$ using $q$-cyclotomic cosets of $n$. This method also includes splittings consisting of quadratic residues and nonresidues as shown below.

Corollary 3.6. (i) If $q$ is a square modulo $n$, then there is a splitting $\left(S_{1}, S_{2}\right)$, each of which consists of a union of $q$-cyclotomic cosets of $n$.

(ii) If $n$ is an odd prime and $q$ is a square modulo $n$, then the set of quadratic residues of $n$ consists of a union of q-cyclotomic cosets of $n$, and so does the set of quadratic nonresidues of $n$.

Proof. The existence of a splitting $\left(S_{1}, S_{2}\right)$, each of which consists of a union of $q$-cyclotomic cosets of $n$, is equivalent to the existence of duadic codes of length $n$ over $\mathbb{F}_{q}$. Part (ii) follows from Theorem [3.5. Part (iii) is an easy exercise in [16, p. 237].

In what follows, we consider 4-cyclotomic cosets of an odd $n$ since $4=2^{2}$ is a square modulo $n$. By Corollary 3.6 (ii), duadic double circulant codes with a splitting $\left(S_{1}, S_{2}\right)$, each of which consists of a union of 4-cyclotomic cosets of $n$ contain Gaborit's quadratic double circulant codes [5] if $n>2$ is a prime.

The total number of splittings which consist of unions of 4-cyclotomic cosets for an odd $n$ such that $3 \leq n \leq 41$ can be obtained from [16, Table 6.1, Table 6.3] and [21]. We compute explicitly splittings for $3 \leq n \leq 41$. We also compute all the splittings which consist of unions of 9-cyclotomic cosets for an odd $n$ such that $5 \leq n \leq 37$. To save space, we give them in [18]. 


\section{Computational results}

We compute all DDC codes of lengths up to $82,82,62,58,38$ over $\mathbb{F}_{2}, \mathbb{F}_{3}, \mathbb{F}_{4}, \mathbb{F}_{5}$, and $\mathbb{F}_{7}$, respectively, based on 4-cyclotomic cosets, and one DDC code over $\mathbb{F}_{3}$ based on 9-cyclotomic cosets. The results are displayed in Table 1 to Table 5. Full tables including all the missing lengths can be found in [18]. We have used Magma [2] whenever it is necessary.

In Table 1, the first column $n$ indicates an odd positive integer in the duadic splitting. The second column "cl" indicates the corresponding code length. The third column SD(I) indicates the maximum minimum distance for self-dual Type I codes in our calculation. The fourth column SD(II) indicates the maximum minimum distance for self-dual Type II codes in our calculation. The fifth column NSD indicates the maximum minimum distance for non self-dual codes in our calculation. The sixth column O.SD(I) indicates the optimal minimum distance for self-dual Type I codes in [6]. The seventh column O.SD(II) indicates the optimal minimum distance for self-dual Type II codes in [6]. The eighth column O.Linear indicates the optimal minimum distance for linear codes in [8]. In the ninth column Comment, $\mathrm{O}(\mathrm{I})$ indicates our construction for self-dual Type I code is optimal, $\mathrm{O}(\mathrm{II})$ indicates our construction for self-dual Type II code is optimal, $\mathrm{O}(\mathrm{L})$ indicates our construction is optimal in linear codes, and $\mathrm{B}(\mathrm{L})$ indicates the maximum minimum distance of our construction is equal to that of the best known linear codes.

In Table 2, O.SD comes from [6]. In Table 3, O.SD $(\mathrm{E})$ and $\mathrm{O} \cdot \mathrm{SD}(\mathrm{H})$ come from [6], [12]. In Table 4, O.SD comes from [6], [12], [14], [15]. In Table 5, O.SD comes from [6], [12], [13]. For all tables, O.Linear comes from [8]. From our DDC construction, we show that there are many optimal self-dual codes or optimal linear codes (or codes which has the best known linear code parameters).

The following examples help readers to understand our construction method. Furthermore, the examples are nontrivial to obtain and connect our codes with other known codes.

Example 4.1. (i) $n=15$ in $\mathbb{F}_{2}$.

With the following splitting

$$
S_{1}=\{1,4,3,12,7,13,5\}, \mu_{2}
$$

$\mathcal{P}_{15}\left(0,0,1, S_{1}, S_{2}\right)$ is a $[30,15,8]$ optimal linear code and $\mathcal{B}_{15}\left(0,1,0,0,0,1, S_{1}, S_{2}\right)$ is a $[32,16,8]$ optimal linear code.

$\mathcal{P}_{15}\left(0,0,1, S_{1}, S_{2}\right)$ is an extremal formally self-dual even code. There are exactly six $[30,15,8]$ extremal double circulant formally self-dual even codes [9]. Since the order of automorphism group for our code is 60 , our code is equivalent to $C_{30,4}$ in [9]. $\mathcal{B}_{15}\left(0,1,0,0,0,1, S_{1}, S_{2}\right)$ is not a formally self-dual code.

(ii) $n=17$ in $\mathbb{F}_{2}$.

With the following spitting

$$
S_{1}=\{1,4,16,13,3,12,14,5\}, \mu_{-2}
$$

$\mathcal{P}_{17}\left(1,0,1, S_{1}, S_{2}\right)$ is a $[34,17,8]$ optimal linear code and $\mathcal{B}_{17}\left(0,1,0,1,0,1, S_{1}, S_{2}\right)$ is a $[36,18,8]$ optimal linear codes. We have checked that with the splitting of quadratic 
residues and quadratic nonresidues of $n=17$, all the minimum distance of DDC codes are less than 8 .

$\mathcal{P}_{17}\left(1,0,1, S_{1}, S_{2}\right)$ is a near-extremal formally self-dual even code. There are exactly five weight distributions for $[34,17,8]$ near-extremal double circulant formally selfdual even codes [9]. Our code has $\alpha=17$ in the notation of weight distribution [9]. $\mathcal{B}_{17}\left(0,1,0,1,0,1, S_{1}, S_{2}\right)$ is not a formally self-dual code.

(iii) $n=33$ in $\mathbb{F}_{2}$.

With the following splitting

$$
S_{1}=\{1,4,16,31,25,3,12,15,27,9,7,28,13,19,10,11\}, \mu_{-1},
$$

we have that $\mathcal{P}_{33}\left(1,0,1, S_{1}, S_{2}\right)$ is a $[66,33,12]$ Type I optimal self-dual code and that $\mathcal{B}_{33}\left(0,1,1,0,0,1, S_{1}, S_{2}\right)$ is a $[68,34,12]$ Type I optimal self-dual code. We also show that $\mathcal{P}_{33}\left(0,0,1, S_{1}, S_{2}\right)$ has the best known parameters $[66,33,12]$.

From [10], there are 3 inequivalent pure double circulant optimal [66, 33, 12] Type I selfdual codes and there are 84 inequivalent bordered double circulant optimal $[68,34,12]$ Type I self-dual codes. Both $\mathcal{P}_{33}\left(1,0,1, S_{1}, S_{2}\right)$ and $\mathcal{B}_{33}\left(0,1,1,0,0,1, S_{1}, S_{2}\right)$ have automorphism group order 330 and the number of minimum codewords 858 so that $\mathcal{P}_{33}\left(1,0,1, S_{1}, S_{2}\right)$ is equivalent to $C_{66,21}$ in [10, Table 3] and $\mathcal{B}_{33}\left(0,1,1,0,0,1, S_{1}, S_{2}\right)$ is equivalent to $C_{68,1}^{\prime}$ in [10, Table 7]. $\mathcal{P}_{33}\left(0,0,1, S_{1}, S_{2}\right)$ is a formally self-dual odd code.

(iv) $n=41$ in $\mathbb{F}_{2}$.

With the following splitting

$$
S_{1}=\{1,4,16,23,10,40,37,25,18,31,3,12,7,28,30,38,29,34,13,11\}, \mu_{-2}
$$

$\mathcal{P}_{41}\left(1,0,1, S_{1}, S_{2}\right)$ has the best known linear code parameters $[82,41,14]$. We have checked that with the splitting of quadratic residue and quadratic nonresidue of $n=17$, all the minimum distance of duadic pure double circulant codes are less than 14 . We recall that $\mathcal{P}_{41}\left(1,0,1, S_{1}, S_{2}\right)$ is a formally self-dual even code.

Example 4.2. $n=43$ in $\mathbb{F}_{2}$. Consider the following splitting

$$
S_{1}:=\{1,4,16,21,41,35,11,3,12,5,20,37,19,33,7,28,26,18,29,30,34\}, \mu_{2},
$$

Then $\mathcal{P}_{43}\left(0,1,0, S_{1}, S_{2}\right)$ is a $[86,43,16]$ optimal Type I self-dual code and the bordered code $\mathcal{B}_{43}\left(0,1,1,1,1,0, S_{1}, S_{2}\right)$ is a $[88,44,16]$ optimal Type II self-dual code. We have checked that using the splitting of quadratic residues and quadratic nonresidues $\mathcal{P}_{43}\left(0,1,0, S_{1}, S_{2}\right)$ is a $[86,43,14]$ Type I self-dual code and $\mathcal{B}_{43}\left(0,1,1,1,1,0, S_{1}, S_{2}\right)$ is a $[88,44,16]$ optimal Type II self-dual code [5]. We have verified that our $\mathcal{P}_{43}\left(0,1,0, S_{1}, S_{2}\right)$ is equivalent to the code in [3], and $\mathcal{B}_{43}\left(0,1,1,1,1,0, S_{1}, S_{2}\right)$ is not equivalent to the code $\mathcal{B}_{43}\left(0,1,1,1,1,0, S_{1}, S_{2}\right)$ with the splitting of quadratic residue and quadratic nonresidue. Note that there exist at least 70 extremal binary doubly-even self-dual codes of length 88 [7]. On the other hand, it is known [11] that there are exactly 151 extremal binary doubly-even self-dual codes of length 88. Hence our code $\mathcal{B}_{43}\left(0,1,1,1,1,0, S_{1}, S_{2}\right)$ will be equivalent to one of them. However these codes [11] were found by an exhaustive computer search and do not give an algebraic structure. 
Example 4.3. As 4-cyclotomic cosets produce good binary DDC codes, it is natural to consider 9-cyclotomic cosets to construct good ternary DDC codes. In fact, we find a new ternary self-dual code which has the best known parameters $[76,38,18]$. The code is $\mathcal{B}_{37}\left(1,1,1,2,0,2, S_{1}, S_{2}\right)$ over $\mathbb{F}_{3}$ with the following splitting.

$$
S_{1}=\{1,9,7,26,12,34,10,16,33,2,18,14,15,24,31,20,32,29\}, \mu_{-1} .
$$

We remark that with the splitting of quadratic residues and quadratic nonresidues of $n=37$, there is no self-dual code. It is known [6] that there is one $[76,38,18]$ ternary self-dual code, denoted by $\left(f_{1} ; 1,35\right)$. We have checked that $\mathcal{B}_{37}\left(1,1,1,2,0,2, S_{1}, S_{2}\right)$ is not equivalent to $\left(f_{1} ; 1,35\right)$. More precisely, our code has the automorphism group of order 1332 and the number of minimum codewords $A_{18}=79032$ while $\left(f_{1} ; 1,35\right)$ has the automorphism group of order 76 and $A_{18}=71136$.

Theorem 4.4. There are at least two ternary self-dual [76,38,18] codes.

\section{Conclusion}

We have introduced a subclass of double circulant codes, called duadic double circulant codes. This is a natural generalization of quadratic double circulant codes [5]. We have constructed many interesting linear (self-dual) codes over $\mathbb{F}_{2}, \mathbb{F}_{3}, \mathbb{F}_{4}, \mathbb{F}_{5}$ and $\mathbb{F}_{7}$. As shown from our tables, many of our codes have good minimum distances. In particular, we have found a new self-dual ternary $[76,38,18]$ code, which is not equivalent to the previously known code with the same parameters in [6].

\section{Acknowledgment}

S. Han was supported by the Basic Science Research Program through the National Research Foundation of Korea (NRF), which is supported by the Ministry of Education, Science and Technology (2010-0007232). J.-L. Kim was partially supported by the Project Completion Grant (year 2011-2012) at the University of Louisville.

\section{References}

[1] R. A. Brualdi,V. Pless, Polyadic codes, Discrete Applied Mathematics, 25 (1989), 317

[2] J. Cannon and C. Playoust, An Introduction to Magma, University of Sydney, Sydney, Australia, 1994, version V2.12-19.

[3] S. T. Dougherty, T. A. Gulliver, and M. Harada, Extremal binary self-dual codes, IEEE Trans. Inform. Theory, 43 (1997), 2036-2047.

[4] S. T. Dougherty, J.-L. Kim, and P. Solé, Double circulant codes from two class association schemes, Advences in Math. of Communications, 1 (2007), 45-64. 
[5] P. Gaborit, Quadratic double circulant codes over fields, J. Combin. Theory, Ser. A, 97 (2002), 85-107.

[6] P. Gaborit and A. Otmani, Experimental constructions of self-dual codes, Finite Fields Appl., 9 (2003), 372-394.

[7] V. Goodwin and V. Yorgov, New extremal self-dual doubly-even binary codes of length 88, Finite Fields Appl., 11 (2005), 1-5.

[8] M. Grassl, Bounds on the minimum distance of linear codes, online available at http://www.codetables.de. Accessed on June 1, 2011.

[9] T. A. Gulliver and M. Harada, Classifiation of extremal double circulant formally selfdual even codes, Des. Codes and Cryptogr. 11 (1997), 25-35.

[10] T. A. Gulliver and M. Harada, Classification of extremal double circulant self-dual codes of lengths 64 to 72, Des. Codes and Cryptogr., 13 (1998), 257-269.

[11] T. A. Gulliver and M. Harada, Classification of extremal double circulant self-dual codes of lengths 74-88, Discrete Math. 306 (2006), 2064-2072.

[12] T. A. Gulliver and M. Harada, New nonbinary self-dual codes, IEEE Trans. Inform. Theory, 54 (2008), 415-417.

[13] T. A. Gulliver, M. Harada, and H. Miyabayashi, Double circulant self-dual codes over $\mathbb{F}_{5}$ and $\mathbb{F}_{7}$, Advances in Math. of Communications, 1 (2007), 223-238.

[14] S. Han and J.-L. Kim, On self-dual codes over $\mathbb{F}_{5}$, Des. Codes and Cryptogr., 48 (2008), $43-58$.

[15] M. Harada and A. Munemasa, There exists no self-dual $[24,12,10]$ code over $\mathbb{F}_{5}$, Des. Codes and Cryptogr., to appear.

[16] W. C. Huffman and V. S. Pless, Fundamentals of Error-Correcting Codes, Cambridge: Cambridge University Press (2003).

[17] M. Karlin, New binary coding results by circulants, IEEE Trans. Inform. Theory, 15 (1969), 81-92.

[18] J.-L. Kim, www.math.louisville.edu/ jlkim/preprints.html.

[19] P. Langevin and P. Solé, Duadic $Z_{4}$-codes, Finite Fields and Their Applications, 6 (2000), 309-326.

[20] S. Ling and C. Xing, Polyadic codes revisited, IEEE Trans. Inform. Theory, 50, No. 1, (2004), 200-207.

[21] V. Pless, Q-codes, J. Combin. Theory, Ser. A, 43 (1986), 258-276. 
Table 1: Duadic double circulant codes over $\mathbb{F}_{2}$

\begin{tabular}{|c|c|c|c|c|c|c|c|l|}
\hline$n$ & $\mathrm{cl}$ & $\mathrm{SD}(\mathrm{I})$ & $\mathrm{SD}(\mathrm{II})$ & $\mathrm{NSD}$ & $\mathrm{O} . \mathrm{SD}(\mathrm{I})$ & $\mathrm{O} . \mathrm{SD}(\mathrm{II})$ & O.Linear & Comment \\
\hline 3 & 6 & 2 & & 3 & 2 & & 3 & $\mathrm{O}(\mathrm{I}), \mathrm{O}(\mathrm{L})$ \\
\hline 3 & 8 & 2 & 4 & 3 & 2 & 4 & 4 & $\mathrm{O}(\mathrm{I}), \mathrm{O}(\mathrm{II}), \mathrm{O}(\mathrm{L})$ \\
\hline 5 & 10 & 2 & & 4 & 2 & & 4 & $\mathrm{O}(\mathrm{I}), \mathrm{O}(\mathrm{L})$ \\
\hline 5 & 12 & 4 & & 4 & 4 & & 4 & $\mathrm{O}(\mathrm{I}), \mathrm{O}(\mathrm{L})$ \\
\hline 7 & 14 & 2 & & 4 & 4 & & 4 & $\mathrm{O}(\mathrm{L})$ \\
\hline 7 & 16 & 2 & 4 & 4 & 4 & 4 & 5 & $\mathrm{O}(\mathrm{II})$ \\
\hline 9 & 18 & 4 & & 4 & 4 & & 6 & $\mathrm{O}(\mathrm{I})$ \\
\hline 9 & 20 & 4 & & 4 & 4 & & 6 & $\mathrm{O}(\mathrm{I})$ \\
\hline 11 & 22 & 6 & & 7 & 6 & & 7 & $\mathrm{O}(\mathrm{I}), \mathrm{O}(\mathrm{L})$ \\
\hline 11 & 24 & 2 & 8 & 7 & 6 & 8 & 8 & $\mathrm{O}(\mathrm{II}), \mathrm{O}(\mathrm{L})$ \\
\hline 13 & 26 & 2 & & 7 & 6 & & 7 & $\mathrm{O}(\mathrm{L})$ \\
\hline 13 & 28 & 4 & & 8 & 6 & & 8 & $\mathrm{O}(\mathrm{L})$ \\
\hline 15 & 30 & 2 & & 8 & 6 & & 8 & $\mathrm{O}(\mathrm{L})$ \\
\hline 15 & 32 & 2 & 4 & 8 & 8 & 8 & 8 & $\mathrm{O}(\mathrm{L})$ \\
\hline 17 & 34 & 2 & & 8 & 6 & & 8 & $\mathrm{O}(\mathrm{L})$ \\
\hline 17 & 36 & 4 & & 8 & 8 & & 8 & $\mathrm{O}(\mathrm{L})$ \\
\hline 19 & 38 & 8 & & 7 & 8 & & $8-9$ & $\mathrm{O}(\mathrm{I}), \mathrm{B}(\mathrm{L})$ \\
\hline 19 & 40 & 2 & 8 & 8 & 8 & 8 & $9-10$ & $\mathrm{O}(\mathrm{II})$ \\
\hline 29 & 58 & 2 & & 12 & 10 & & $12-14$ & $\mathrm{O}(\mathrm{L})$ \\
\hline 29 & 60 & 4 & & 12 & 12 & & $12-14$ & $\mathrm{O}(\mathrm{L})$ \\
\hline 33 & 66 & 12 & & 12 & 12 & & $12-16$ & $\mathrm{O}(\mathrm{I}), \mathrm{B}(\mathrm{L})$ \\
\hline 33 & 68 & 12 & & 12 & 12 & & $13-16$ & $\mathrm{O}(\mathrm{I})$ \\
\hline 41 & 82 & 2 & & 14 & $14-16$ & & $14-20$ & $\mathrm{~B}(\mathrm{~L})$ \\
\hline
\end{tabular}

[22] V. Pless, Symmetry codes over GF(3) and new five-designs, J. Comb. Theory, Ser. A, 12 (1972), 119-142

[23] V. Pless, J. J. Rushanan, Triadic codes, Linear Algebra and its Applications, 98, (1988), 415-433 
Table 2: Duadic double circulant codes over $\mathbb{F}_{3}$

\begin{tabular}{|c|c|c|c|c|c|c|}
\hline$n$ & cl & SD & NSD & O.SD & O.Linear & Comment \\
\hline 3 & 6 & & 3 & & 3 & $\mathrm{O}(\mathrm{L})$ \\
\hline 3 & 8 & & 4 & 3 & 4 & $\mathrm{O}(\mathrm{L})$ \\
\hline 5 & 10 & & 5 & & 5 & $\mathrm{O}(\mathrm{L})$ \\
\hline 5 & 12 & 6 & 5 & 6 & 6 & $\mathrm{O}(\mathrm{S}), \mathrm{O}(\mathrm{L})$ \\
\hline 7 & 16 & 6 & 6 & 6 & 6 & $\mathrm{O}(\mathrm{S}), \mathrm{O}(\mathrm{L})$ \\
\hline 11 & 22 & & 8 & & 8 & $\mathrm{O}(\mathrm{L})$ \\
\hline 11 & 24 & 9 & 8 & 9 & 9 & $\mathrm{O}(\mathrm{S}), \mathrm{O}(\mathrm{L})$ \\
\hline 13 & 26 & & 8 & & $8-9$ & $\mathrm{~B}(\mathrm{~L})$ \\
\hline 17 & 34 & & 11 & & $11-12$ & $\mathrm{~B}(\mathrm{~L})$ \\
\hline 17 & 36 & 12 & 11 & 12 & 12 & $\mathrm{O}(\mathrm{S}), \mathrm{O}(\mathrm{L})$ \\
\hline 19 & 38 & & 11 & & $11-13$ & $\mathrm{O}(\mathrm{L})$ \\
\hline 19 & 40 & 12 & 11 & 12 & $12-14$ & $\mathrm{O}(\mathrm{S}), \mathrm{B}(\mathrm{L})$ \\
\hline 23 & 46 & & 14 & & $14-15$ & $\mathrm{~B}(\mathrm{~L})$ \\
\hline 23 & 48 & 15 & 14 & 15 & $15-16$ & $\mathrm{O}(\mathrm{S}), \mathrm{B}(\mathrm{L})$ \\
\hline 29 & 58 & & 17 & & $17-19$ & $\mathrm{~B}(\mathrm{~L})$ \\
\hline 29 & 60 & 18 & 17 & 18 & $18-20$ & $\mathrm{O}(\mathrm{S}), \mathrm{B}(\mathrm{L})$ \\
\hline 31 & 62 & & 17 & & $17-20$ & $\mathrm{~B}(\mathrm{~L})$ \\
\hline 31 & 64 & 18 & 17 & 18 & $18-21$ & $\mathrm{O}(\mathrm{S}), \mathrm{B}(\mathrm{L})$ \\
\hline 41 & 82 & & 20 & & $20-26$ & $\mathrm{~B}(\mathrm{~L})$ \\
\hline
\end{tabular}

Table 3: Duadic double circulant codes over $\mathbb{F}_{4}$

\begin{tabular}{|c|c|c|c|c|c|c|c|c|}
\hline$n$ & $\mathrm{cl}$ & $\mathrm{SD}(\mathrm{E})$ & $\mathrm{SD}(\mathrm{H})$ & $\mathrm{NSD}$ & $\mathrm{O} . \mathrm{SD}(\mathrm{E})$ & $\mathrm{O} . \mathrm{SD}(\mathrm{H})$ & O.Linear & Comment \\
\hline 3 & 6 & 2 & 4 & 3 & 3 & 4 & 4 & $\mathrm{O}(\mathrm{H}), \mathrm{O}(\mathrm{L})$ \\
\hline 3 & 8 & 4 & 4 & 4 & 4 & 4 & 4 & $\mathrm{O}(\mathrm{E}), \mathrm{O}(\mathrm{H}), \mathrm{O}(\mathrm{L})$ \\
\hline 5 & 10 & 2 & 4 & 4 & 4 & 4 & 5 & $\mathrm{O}(\mathrm{H})$ \\
\hline 5 & 12 & 4 & 4 & 4 & 6 & 4 & 6 & $\mathrm{O}(\mathrm{H})$ \\
\hline 7 & 14 & 6 & 4 & 6 & 6 & 6 & 6 & $\mathrm{O}(\mathrm{E}), \mathrm{O}(\mathrm{L})$ \\
\hline 7 & 16 & 6 & 4 & 6 & 6 & 6 & 7 & $\mathrm{O}(\mathrm{E})$ \\
\hline 11 & 22 & 6 & 8 & 7 & 8 & 8 & $8-9$ & $\mathrm{O}(\mathrm{H}), \mathrm{B}(\mathrm{L})$ \\
\hline 11 & 24 & 8 & 8 & 8 & $8-10$ & 8 & 9 & $\mathrm{~B}(\mathrm{E}), \mathrm{O}(\mathrm{H})$ \\
\hline 17 & 34 & 2 & 10 & 11 & $10-12$ & $10-12$ & $11-13$ & $\mathrm{~B}(\mathrm{H}), \mathrm{B}(\mathrm{L})$ \\
\hline 17 & 36 & 4 & 12 & 11 & $11-14$ & $12-14$ & $12-14$ & $\mathrm{~B}(\mathrm{H}), \mathrm{B}(\mathrm{L})$ \\
\hline 23 & 46 & 14 & 4 & 14 & $14-16$ & $14-16$ & $14-17$ & $\mathrm{~B}(\mathrm{E}), \mathrm{B}(\mathrm{L})$ \\
\hline 23 & 48 & 14 & 4 & 14 & $14-18$ & $14-18$ & $14-18$ & $\mathrm{~B}(\mathrm{E}), \mathrm{B}(\mathrm{L})$ \\
\hline 31 & 62 & 16 & 4 & 16 & $16-23$ & $18-22$ & $18-23$ & $\mathrm{~B}(\mathrm{E})$ \\
\hline
\end{tabular}


Table 4: Duadic double circulant codes over $\mathbb{F}_{5}$

\begin{tabular}{|c|c|c|c|c|c|c|}
\hline$n$ & $\mathrm{cl}$ & $\mathrm{SD}$ & NSD & O.SD & O.Linear & Comment \\
\hline 3 & 6 & 4 & 4 & 4 & 4 & $\mathrm{O}(\mathrm{S}), \mathrm{O}(\mathrm{L})$ \\
\hline 3 & 8 & 4 & 4 & 4 & 4 & $\mathrm{O}(\mathrm{S}), \mathrm{O}(\mathrm{L})$ \\
\hline 5 & 10 & 2 & 5 & 4 & 5 & $\mathrm{O}(\mathrm{L})$ \\
\hline 5 & 12 & 4 & 6 & 6 & 6 & $\mathrm{O}(\mathrm{L})$ \\
\hline 7 & 14 & 6 & 6 & 6 & 6 & $\mathrm{O}(\mathrm{S}), \mathrm{O}(\mathrm{L})$ \\
\hline 7 & 16 & 7 & 7 & 7 & 7 & $\mathrm{O}(\mathrm{S}), \mathrm{O}(\mathrm{L})$ \\
\hline 11 & 22 & 7 & 8 & 8 & $8-10$ & $\mathrm{~B}(\mathrm{~L})$ \\
\hline 11 & 24 & 9 & 9 & 9 & $9-10$ & $\mathrm{O}(\mathrm{S}), \mathrm{B}(\mathrm{L})$ \\
\hline 17 & 34 & 4 & 11 & $11-12$ & $11-14$ & $\mathrm{~B}(\mathrm{~L})$ \\
\hline 17 & 36 & 4 & 12 & $12-13$ & $12-15$ & $\mathrm{~B}(\mathrm{~L})$ \\
\hline 19 & 38 & 12 & 12 & $12-14$ & $12-16$ & $\mathrm{~B}(\mathrm{~S}), \mathrm{B}(\mathrm{L})$ \\
\hline 19 & 40 & 13 & 13 & $13-15$ & $13-17$ & $\mathrm{~B}(\mathrm{~S}), \mathrm{B}(\mathrm{L})$ \\
\hline 23 & 46 & 14 & 14 & $14-20$ & $14-20$ & $\mathrm{~B}(\mathrm{~S}), \mathrm{B}(\mathrm{L})$ \\
\hline 23 & 48 & 14 & 15 & $14-20$ & $15-20$ & $\mathrm{~B}(\mathrm{~S}), \mathrm{B}(\mathrm{L})$ \\
\hline 29 & 58 & 16 & 17 & $16-24$ & $18-24$ & $\mathrm{~B}(\mathrm{~S})$ \\
\hline
\end{tabular}

Table 5: Duadic double circulant codes over $\mathbb{F}_{7}$

\begin{tabular}{|c|c|c|c|c|c|c|}
\hline$n$ & $\mathrm{cl}$ & $\mathrm{SD}$ & NSD & O.SD & O.Linear & Comment \\
\hline 3 & 6 & & 4 & & 4 & $\mathrm{O}(\mathrm{L})$ \\
\hline 3 & 8 & 5 & 5 & 5 & 5 & $\mathrm{O}(\mathrm{S}), \mathrm{O}(\mathrm{L})$ \\
\hline 5 & 10 & & 5 & & 5 & $\mathrm{O}(\mathrm{L})$ \\
\hline 5 & 12 & 6 & 6 & 6 & 6 & $\mathrm{O}(\mathrm{S}), \mathrm{O}(\mathrm{L})$ \\
\hline 7 & 16 & & 7 & 7 & 7 & $\mathrm{O}(\mathrm{L})$ \\
\hline 11 & 22 & & 9 & & $9-10$ & $\mathrm{~B}(\mathrm{~L})$ \\
\hline 11 & 24 & 9 & 9 & $9-11$ & $10-11$ & $\mathrm{~B}(\mathrm{~S})$ \\
\hline 13 & 26 & & 10 & & $10-12$ & $\mathrm{~B}(\mathrm{~L})$ \\
\hline 13 & 28 & 10 & 10 & $11-13$ & $11-13$ & $\mathrm{~B}(\mathrm{~S})$ \\
\hline 17 & 34 & & 12 & & $12-15$ & $\mathrm{~B}(\mathrm{~L})$ \\
\hline 19 & 38 & & 13 & & $13-17$ & $\mathrm{~B}(\mathrm{~L})$ \\
\hline
\end{tabular}

